\title{
The prevalence and type of pulmonary involvement in ankylosing spondylitis
}

\author{
Nader Rezaei ${ }^{1}$, Simin Almasi ${ }^{2 *}$, Kazem Zamani ${ }^{3}$, Ali Khalooei ${ }^{4}$ \\ ${ }^{1}$ Assistant Professor of Pulmonary Medicine, Department of Pulmonary Medicine, Firouzgar Hospital, Iran University of Medical \\ Science, Tehran, Iran; ${ }^{2}$ Assistant Professor of Rheumatology, Department of Rheumatology, Firouzgar Hospital, Iran University of \\ Medical Sciences, Tehran, Iran ; ${ }^{3}$ Internal Medicine, Department of Rheumatology, Firouzgar Hospital, Iran University of Medical \\ Science, Tehran, Iran; ${ }^{4}$ Assistant Professor of Community Medicine, Department of Community Medicine, School of Medicine, \\ Kerman University of Medical Sciences, Kerman, Iran
}

\begin{abstract}
Ankylosing spondylitis (AS) is a chronic and inflammatory disease that affects the spinal column and peripheral joints. Pulmonary manifestations in patients with ankylosing spondylitis are rare and usually asymptomatic and include restrictions from fibrobullous apical disease, chest wall disease, spontaneous pneumothorax, and obstructive sleep apnea. This crosssectional study was performed on 60 patients with AS. All patients fulfilled the modified New York criteria, 1984. Bath Ankylosing Spondylitis Disease Activity Index (BASDAI) and Bath Ankylosing Spondylitis Functional Index (BASFI), CRP, and ESR were measured in all patients. All patients were visited by a rheumatologist and a pulmonologist, and pulmonary function test (PFT) and chest X-ray (CXR) were performed. High-resolution computed tomography (HRCT) was performed only in patients who had abnormal results in clinical examination, PFT, or CXR. Overall, 50 patients had normal CXR (83.3\%) and 10 patients (16.7\%) had abnormal CXR, including reticulonodular (3.3\%), atelectasis (3.3\%), and lucency (3.3\%). Of the 14 cases that were HRCT, only 8 (57.1\%) had abnormal lung CT scans. The Pulmonary Function Test (PFT) was taken from all patients, and 15 (25\%) patients had abnormal results, $11(18.3 \%)$ had a restrictive pattern, and $4(6.7 \%)$ had an obstructive pattern. The incidence of lung involvement in patients with AS in this study is similar to that of other studies $(16 \%)$; in most cases, the lung involvement is of the restrictive pattern. Lung involvement in these patients may be asymptomatic.
\end{abstract}

Keywords: ankylosing spondylitis, pulmonary manifestation, restrictive pattern.

\section{Introduction}

Ankylosing spondylitis (AS) is a chronic and inflammatory disease that primarily affects the axial skeleton, the entheses, and, less frequently, the peripheral joints. The most prevalent symptom of AS is chronic low back pain, and clinical manifestations usually include limited spinal range of motion, predominantly symmetric bilateral sacroiliitis on radiography, and positive serology for HLA-B27 in 90-95\% of cases [1-3].

Spondylitis can be associated with extra-articular findings, particularly uveitis, and less commonly, cardiac and pulmonary disease [4-6]. Pulmonary disease can develop as a result of restrictive changes caused by the musculoskeletal disease and changes in the lungs themselves, including interstitial, nodular, and parenchymal abnormalities [6,7]. AS occasionally affects the cricoarytenoid joint, and patients can experience hoarseness, sore throat, upper airway obstruction, or respiratory failure. Furthermore, some patients present with other pulmonary changes such as apical pulmonary fibrosis through conventional radiography. Apical fibrosis is usually asymptomatic and in association with a long duration of disease [8-11]. Although high-resolution computed tomography (HRCT) provides a good diagnostic method for the detection of pulmonary abnormalities [11], the exact prevalence and type of such manifestations remain unknown.

Considering the significance of detecting pulmonary involvement as soon as possible, estimating the prevalence and determining the type of such abnormalities are very important. This single center crosssectional study was undertaken to provide data on the prevalence and type of AS pulmonary manifestations.

In these patients, lung involvement can have a poor prognosis, and abnormal lung parenchyma causes superinfection with a number of organisms, such as

\footnotetext{
* Corresponding Author: Simin Almasi, E-mail: Simin_almasi@yahoo.com, Tel: +98 21 82141301, Fax: +98 2166404377
}

Received: 03 September 2017; Accepted: 16 October 2017 
Pulmonary involvement in ankylosing spondylitis

aspergillus or mycobacterium tuberculosis [12,13].

There are no treatments for fibrobullous diseases in ankylosing spondylitis patients, and there are no similar opinions about the therapeutic effects of anti-TNF drugs in pulmonary involvement [14].

\section{Materials and Methods}

\section{Patients and settings}

This is a cross-sectional study, and cases were selected from the registry system of the ankylosing spondylitis patients in Firouzgar Hospital in Tehran, Iran.

Diagnostic criteria for all patients included the diagnostic criteria from the 1984 modified New York criteria for AS [15]. Patients that attended periodic visits were entered into the study.

Informed written consent was obtained from all patients prior to enrollment. Patients were excluded if they had history of asthma or COPD.

All data related to patients was collected over 5-6 months and included: age, gender, HLA-B27 status, bath ankylosing spondylitis disease activity index (BASDAI), bath ankylosing spondylitis functional index (BASFI), Creactive protein (CRP), ESR, duration of disease, and smoking history.

BASDAI was used to determine disease severity. If BASDAI was less than 4 , the disease was considered inactive; if it was equal to or more than 4 , the disease was considered active. All patients were examined, and chest X-ray (CXR) and pulmonary function test (PFT) were performed; if the physical examination, CXR, or PFT results were abnormal, HRCT was performed.

All lung CT scans, CXR, and PFT were evaluated by a pulmonologist, and all results were collected in the provided forms.

This study could have been improved by including a larger number of patients, but the strength of this study is in the patient selection. All patients in this study fulfilled the 1984 modified New York criteria for AS, and, as is known, the specificity of this criteria is very high for the diagnosis of this disease. Therefore, the number of patients was low.

All patients in this study had a high probability of ankylosing spondylitis, but not any other spondyloarthropathy.

If other diagnostic criteria had been used for patients, it is possible that not all studied patients had ankylosing spondylitis, and the amount of bias would have been minimized. It should be noted that the Ethics Committee reviewed and approved this study.

\section{Statistical analysis}

After data was collected, it was entered into the SPSS version 16 software with T-TEST and Nonparametric test (one sample Kolmogorov-Smirnov test). Continuous data was shown as mean \pm standard deviation (SD), and categorical data as numbers with percentages (n, \%), and cross tabs were analyzed according to the requirements of information.

\section{Results}

This study was conducted on $60(\mathrm{M} / \mathrm{F}=51 / 9)$ patients with AS. Mean age was $38.7 \pm 10.7$, with the youngest being 22 years old and the oldest 65 years old. Disease duration was $10.29 \pm 5.8$ years, ESR was $24.1 \pm 20.6$, CRP was $8.63 \pm 15.2$, BASDAI was $3 \pm 1.8$ (BASDAI $<4$ inactive diseases and $\mathrm{BASDAI} \geq 4$ active diseases), and BASFI was $2.3 \pm 1.8$. Other characteristics are presented in Table 1 . This study was conducted in accordance with the ethical principles outlined in the World Medical Association Declaration of Helsinki.

Table 1. Characteristic of patients

\begin{tabular}{ll}
\hline Variable & N/\% \\
\hline age $^{1}$ & $38.7 \pm 10.7$ \\
sex $^{2}(\mathrm{M} / \mathrm{F})$ & $51 / 9(85 \% / 15 \%)$ \\
cigar $^{2}$ & $20(33.3 \%)$ \\
Active diseases $^{2}$ & $19(37.3 \%)$ \\
Positive HLA- B272 $^{2}$ & $31(51.7 \%)$ \\
BASDAI score & $3 \pm 1.8$ \\
BASFI score & $2.3 \pm 1.8$ \\
Duration of diseases $^{1}$ & $10.29 \pm 5.8$ \\
ESR(Erythrocyte sedimentation rate) $^{1}$ & $24.1 \pm 20.6$ \\
CRP(C-Reactive protein) & \\
\hline
\end{tabular}

1 are presented as mean (SD)

2 are presented as $\mathrm{N}(\%)$

Twenty patients were smokers (33.3\%), and all patients were consuming less than one pack per year. Chest X-rays were taken from all patients. Ten patients had abnormalities on CXR, including reticular pattern, atelectasis, nonspecific lucency, thickening of the pleural, and elevated hemidiaphragm (Table 2).

Table 2. Frequency of radiographic findings

\begin{tabular}{ll}
\hline Variable & N/\% \\
\hline Normal & $50(83.3 \%)$ \\
Reticular pattern & $2(3.3 \%)$ \\
Atelectasia & $2(3.3 \%)$ \\
Nonspecific lucency & $2(3.3 \%)$ \\
Thickening of pleural & $2(3.3 \%)$ \\
Elevated hemidiaphragm & $2(3.3 \%)$ \\
\hline
\end{tabular}


Rezaei et al.

In this study, PFT was taken from all patients, and 15 (25\%) patients had abnormal PFT, 11 (18.3\%) patients had a restrictive pattern, and $4(6.7 \%)$ patients had an obstructive pattern.

Lung CT scans were taken of 14 patients and the results are shown in Table 3 .

Table 3. Frequency of HRCT findings

\begin{tabular}{ll}
\hline Variable & N/\% \\
\hline Normal & $4(6.7 \%)$ \\
Noduls & $2(3.3 \%)$ \\
Mosaic pattern & $2(3.3 \%)$ \\
Ground glass & $2(3.3 \%)$ \\
Thickening of pleural & $2(3.3 \%)$ \\
Atelectasis & $1(1.7 \%)$ \\
Emphysema & $2(3.3 \%)$ \\
\hline
\end{tabular}

\section{Discussion}

Ankylosing spondylitis (AS) is one of the families of spondyloarthropathy; there is a direct correlation between the prevalence of the human leukocyte antigen HLA-B27 and ankylosing spondylitis.

Reports of pulmonary disease in these patients is not high; however, it seems that many of these patients are asymptomatic.

HRCT abnormalities in these patients that have been reported include unilateral or bilateral apical fibrosis, emphysema, thickening of the pleura and pleural disease, interstitial lung disease (ILD), and mycetoma.

Kiris et al. studied 28 patients. For all patients, chest $\mathrm{X}$-ray, pulmonary function test, and HRCT were performed. All patients had normal chest X-rays; 18 $(64.28 \%)$ patients had abnormal HRCT, including mosaic pattern (35.7\%), subpleural nodule $(25 \%)$, and parenchymal band (17.8\%) [16].

In the current study, HRCT was performed in only 14 (23.3\%) patients, for ethical issues. Among these, 8 $(57.1 \%)$ patients had abnormality in HRCT scan. Although not all patients in this study had an HRCT scan, for those patients who were symptomatic in history, physical exam, or had an abnormality in CXR or PFT, HRCT was performed.

Of the patients who had CT scan, only $6(42.9 \%)$ had an active disease. There is no statistically significant relationship between disease activity and HRCT scan $(p=0.56)$.

In these patients, the only statistically significant association was found between gender $(p=0.01)$ and CRP $(p=0.01)$ and CT findings. Complete patient information is presented in Table 4.
Table 4. Characteristics of the patients who became the CT lung

\begin{tabular}{lll}
\hline Variable & N/\% & p-value \\
\hline Normal/ab Normal & $6(42.9 \%) / 8(57.1 \%)$ & \\
M/F & $13(92.9 \%) / 1(7.1 \%)$ & 0.01 \\
Age & $39.7 \pm 1.36$ & 0.09 \\
CRP & $10.9 \pm 1.56$ & 0.01 \\
ESR & $18.7 \pm 1.56$ & 0.4 \\
HLA-B27 & $4(28.6 \%)$ & 0.1 \\
BASDAI & $3.5 \pm 2.2$ & \\
Activity & $6(42.9 \%)$ & 0.56 \\
Duration & $11 \pm 3.8$ & 0.6 \\
Cigar & $6(42.9 \%)$ & 0.3 \\
\hline
\end{tabular}

HRCT was not requested for all patients, and this is a challenge in the current study. The Ethics Committee did not allow HRCT to be taken in asymptomatic patients.

In this study, $10(16.6 \%)$ patients had an abnormality in CXR; the most abnormal changes included reticular (3.3\%) and nodular (3.3\%) patterns. Only 2 (3.3\%) patients had normal CXR and abnormal HRCT. In Kiris et al. (16), all 28 patients with AS had a normal CXR, and 18 of these patients had an abnormal HRCT. This result differs from the results of the current study. In this study, 7 out of 10 patients who had abnormal CXR were smokers, and 13 out of 50 patients who had normal CXR were smokers. The mean disease duration was 9.4 years in patients who had abnormal CXR and 10.5 years in patients with normal CXR. Thus, patients who were smokers had more abnormal changes in CXR $(p=0.05)$.

The Pulmonary Function Test (PFT) was performed for all patients; 15 (25\%) patients showed abnormal PFT, $11(18.3 \%)$ patients showed a restrictive pattern, and 4 (6.7\%) patients had an obstructive pattern. Of the 15 patients who had abnormal PFT, only 5 of them were smokers. No significant association between smoking and abnormal PFT (p-value=0.38) was found. The mean of BASFI in patients who have had a restrictive pattern was 3.1 , and there is a significant association between BASFI and restrictive pattern in these patients $(p=0.005)$. In Berdal et al., as in the current study, showed that most patients with a restrictive pattern had reduced range of motion in the spine [17]. Thus, preventing structural deformity can be prevented in these patients, it would be a great help to our patients. It seems that in ankylosing spondylitis (AS), the prevalence of pulmonary involvement is more particularly a restrictive pattern.

Berdal et al. (2012) showed significantly impaired pulmonary function in AS patients compared to the controls and demonstrated a clear relationship between reduced spinal mobility and restrictive PF in AS patients [17]. 


\section{Conclusion}

This study showed that the incidence of lung involvement in patients with AS is similar to that of other studies $(16 \%)$, and in most cases, lung involvement is of the restrictive pattern. Lung involvement in these patients may be asymptomatic; thus, it seems that performing PFT and CXR would be better for all patients with AS, even if they have not had symptoms of respiratory involvement,
PFT, or CXR.

\section{Conflicts of interest}

The authors declare no conflict of interest.

\section{Acknowledgments}

The authors would like to thank the patients who participated in this study.

\section{References}

1. Lawrence RC, Helmick CG, Arnett FC, Deyo RA, Felson DT, Giannini $\mathrm{EH}$, et al. Estimates of the prevalence of arthritis and selected musculoskeletal disorders in the United States. Arthritis \& Rheumatism 1998; 41(5): 778-99. doi: 10.1002/1529-0131(199805)41: 5<778::AID-ART4>3.0.CO;2-V.

2. Braun J, Bollow M, Remlinger G, Eggens U, Rudwaleit M, Distler A, et al. Prevalence of spondylarthropathies in HLA-B27 positive and negative blood donors. Arthritis \& Rheumatism 1998; 41(1): 58-67. doi: 10.1002/15290131(199801)41:1<58::AID-ART8> 3.0.CO;2-G.

3. Ng SC, Liao Z, Yu DTT, Chan ESY, Zhao $\mathrm{L}, \mathrm{Gu}$ J, editors. Epidemiology of spondyloarthritis in the People's Republic of China: review of the literature and commentary. Seminars in Arthritis and Rheumatism 2007: Elsevier. doi: 10.1016/j.semarthrit.2007.01. 003.

4. Baron M, Zendel I. HLA-B27 testing in ankylosing spondylitis: an analysis of the pretesting assumptions. The Journal of Rheumatology 1989; 16(5): 631-4; discussion 4-6.

5. Franquet $\mathrm{T}$, Müller N, Flint J. A patient with ankylosing spondylitis and recurrent haemoptysis. European Respiratory Journal 2004; 23(3): 488-9. doi: 10.1183/ 09031936.04.00042804.

6. Casserly I, Fenlon H, Breatnach E, Sant S. Lung findings on highresolution computed tomography in idiopathic ankylosing spondylitis-correlation with clinical findings, pulmonary function testing and plain radiography. Rheumatology 1997;

36(6): 677-82, doi: 10.1093/ rheumatology/36.6.677.

7. Solak Ö, Fidan F, Dündar Ü, Türel A, Ayçiçek A, Kavuncu V, et al. The prevalence of obstructive sleep apnoea syndrome in ankylosing spondylitis patients. Rheumatology 2009; 48(4): 433-5. doi: 10.1093/ rheumatology/kep021.

8. El Maghraoui A, Chaouir S, Abid A, Bezza A, Tabache F, Achemlal L, et al. Lung findings on thoracic highresolution computed tomography in patients with ankylosing spondylitis. Correlations with disease duration, clinical findings and pulmonary function testing. Clinical Rheumatology 2004; 23(2): 123-8. doi: 10.1007/s10067-003-0845-8.

9. Sampaio-Barros PD, Cerqueira EMF, Rezende SM, Maeda L, Conde RA, Zanardi VA, et al. Pulmonary involvement in ankylosing spondylitis. Clinical Rheumatology 2007; 26(2): 225-30. doi: 10.1007/s10067-006-0286-2.

10. Lee CC, Lee SH, Chang IJ, Lu TC, Yuan A, Chang TA, et al. Spontaneous pneumothorax associated with ankylosing spondylitis. Rheumatology. 2005; 44(12): 1538-41. doi: 10.1093/ rheumatology/kei077.

11. Ayhan-Ardic FF, Oken O, Yorgancioglu ZR, Ustun N, Gokharman FD. Pulmonary involvement in lifelong nonsmoking patients with rheumatoid arthritis and ankylosing spondylitis without respiratory symptoms. Clinical Rheumatology. 2006; 25(2): 213-8. doi: 10.1007/s10067005-1158-x.

12. Tan CK, Lai CC, Chou CH, Hsueh
POR. Mycobacterium celatum pulmonary infection mimicking pulmonary tuberculosis in a patient with ankylosing spondylitis, International Journal of Infectious Diseases 2009; 13(6): e459-e462, doi: 10.1016/j.ijid.2008.12.014.

13. Kennedy WPU, Milne LJ, Blyth W, Crompton GK. Two unusual organisms, Aspergillus terreus and Metschnikowia pulcherrima, associated with the lung disease of ankylosing spondylitis. Thorax 27(5): 604-610, doi: 10.1136/thx.27. 5.604 .

14. Kanathur NL, Lee-Chiong $\mathrm{T}$. Pulmonary manifestations of ankylosing spondylitis. Clin Chest Med 2010 Sep; 31(3): 547-54. doi: 10.1016/j.ccm.2010.05.002.

15. Linden SVD, Valkenburg HA, Cats A. Evaluation of diagnostic criteria for ankylosing spondylitis. Arthritis \& Rheumatology 1984; 27(4): 3618. doi: 10.1002/art.1780270401.

16. Kiris A, Ozgocmen S, Kocakoc E, Ardicoglu O, Ogur E. Lung findings on high resolution $\mathrm{CT}$ in early ankylosing spondylitis. European Journal of Radiology 2003; 47(1): 71-6, doi: 10.1016/s0720-048x(02) 00085-2.

17. Berdal G, Halvorsen $S$, van der Heijde D, Mowe M, Dagfinrud H. Restrictive pulmonary function is more prevalent in patients with ankylosing spondylitis than in matched population controls and is associated with impaired spinal mobility: a comparative study. Arthritis Research \& Therapy 2012; 14(1): R19, doi: 10.1186/ ar3699. 